\title{
Metabolism of fibrates by cytochrome P450s and UDP- glycosyltransferases in rat and human liver microsomes
}

\author{
ZHOU YanMi ${ }^{1,2^{*}}$, LIU AiMing ${ }^{3 *}$, XIE Hui ${ }^{2}$, CHENG Gary $Q^{4} \&$ DAI RenKe $e^{1,2 \dagger}$ \\ ${ }^{1}$ School of Bioscience and Bioengineering, South China University of Technology, Guangzhou 510006, China; \\ ${ }^{2}$ Guangzhou Institute of Biomedicine and Health, Chinese Academy of Sciences, Guangzhou 510530, China; \\ ${ }^{3}$ Medical School of Ningbo University, Ningbo 315211, China; \\ ${ }^{4}$ South China Center for Innovative Pharmaceuticals, Guangzhou 510006, China
}

Received March 17, 2011; accepted September 9, 2011; published online February 15, 2012

\begin{abstract}
Fibrates are widely used for the treatment of dyslipidemia. However, the contributions of the phase I and phase II metabolic pathways to the clearance of fibrates are unclear. In this study, we investigated the metabolism of gemfibrozil (Gem), clofibric acid (CA), fenofibric acid (FA) and bezafibrate (Beza) by cytochrome P450s (P450s) and UDP-glycosyltransferases (UGTs) using a substrate depletion approach. We also compared the metabolic characteristics of rat liver microsomes (RLM) and human liver microsomes (HLM). The intrinsic clearance rates mediated by P450s, UGTs and both were $172 \pm 22,643 \pm 26,798 \pm 103 \mu \mathrm{L}$ $\mathrm{min}^{-1} \mathrm{mg}^{-1}$, respectively, for Gem and $43 \pm 11,88 \pm 12,119 \pm 15 \mu \mathrm{L} \mathrm{min}{ }^{-1} \mathrm{mg}^{-1}$, respectively, for CA in RLM. The fractions metabolized by P450s and UGTs in RLM were $22 \%$ and $81 \%$ for Gem, $36 \%$ and $74 \%$ for CA. The P450- and UGT-mediated depletion rates for Gem were 303 and $1607 \mathrm{nmol} \mathrm{min} \mathrm{mg}^{-1}$ in RLM versus 86 and $243 \mathrm{nmol} \mathrm{min}^{-1} \mathrm{mg}^{-1}$ in HLM. The corresponding rates for CA were 1.1 and $1.7 \mathrm{nmol} \mathrm{min}{ }^{-1} \mathrm{mg}^{-1}$ in RLM versus 0.025 and $0.038 \mathrm{nmol} \mathrm{min}^{-1} \mathrm{mg}^{-1}$ in HLM. Accordingly, both P450s and UGTs substantially contribute to the clearance of Gem and CA, with UGTs playing a greater role. To avoid under-estimating the impact of these pathways, it is necessary to measure NADPH- and UDPGA-dependent metabolism. Although the fractions of these two pathways in RLM and HLM were similar, the depletion rate of Gem and CA in RLM was higher than that in HLM. The metabolism of FA and Beza by P450s and UGTs was too low to calculate intrinsic clearance in both RLM and HLM. These results indicate that fibrates are metabolized via similar pathways in rats and humans, and it is applicable to use RLM to predict the clearance of fibrates in human.
\end{abstract}

cytochrome P450, UDP-glycosyltransferase, fibrates, metabolism, microsomes

Citation: Zhou Y M, Liu A M, Xie H, et al. Metabolism of fibrates by cytochrome P450s and UDP-glycosyltransferases in rat and human liver microsomes. Chin Sci Bull, 2012, 57: 1142-1149, doi: 10.1007/s11434-011-4958-1

Fibrates have been used for decades to treat dyslipidemia. Clofibrate and fenofibrate are prodrugs that are hydrolyzed to clofibric acid (CA) and fenofibric acid (FA) in vivo [1-3]. In contrast, gemfibrozil (Gem) and bezafibrate (Beza) are active compounds. The chemical structures of these four fibrates are shown in Figure 1. Although fibrates were originally thought to be safe in clinical use, they have been shown to cause species-related carcinogenesis in rodents, to which humans seem to be refractory [4-6]. However, severe

*These authors contributed equally to this work.

†Corresponding author (email: rdai@scut.edu.cn) myotoxicity associated with fibrates, particularly with Gem, has been reported in clinical use [7,8], where metabolic drug-drug interactions may play an important role [9]. The mechanisms responsible for these toxicities are currently under investigation. Detailed metabolic studies of fibrates may help us to better understand the mechanisms involved in these toxicities and in their potential drug-drug interactions.

The metabolism of fibrates has been investigated in several species using in vivo and in vitro methods $[10,11]$. Gem is extensively metabolized and only about $5 \%$ of parent compound is retained. Its main metabolites include acyl 


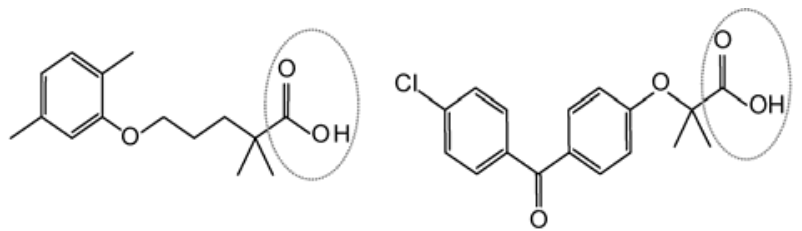

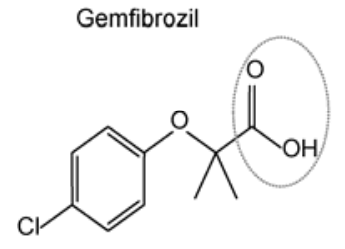

Clofibric acid

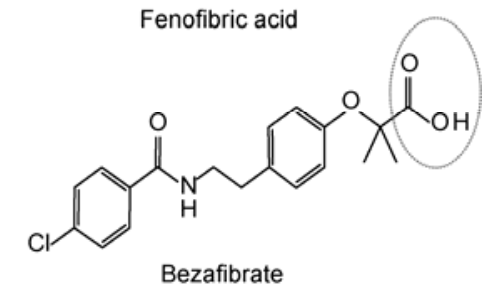

Figure 1 Chemical structures of fibrates showing similar scaffolds. The carboxyl groups are circled.

glucuronide conjugates, accounting for $32 \%$ of all of Gem's metabolites in urine after administration [12-14]. For CA, $60 \%$ of the dose is conjugated as glucuronide metabolites in humans [15]. As the principal metabolite of fenofibrate, FA is excreted either unchanged or as glucuronide conjugates in rat and human urine [16]. About half of Beza recovered in urine was unchanged, with the remainder excreted as glucuronide conjugates $(22 \%)$ and polar metabolites $(22 \%)$ in humans [17]. However, there are contrary opinions regarding the relative contribution of phase I metabolism to the metabolic clearance of fibrates, either neglecting it [11] or considering that fibrates were principally metabolized by P450 3A4 [10]. To determine the relative contribution of oxidation and glucuronidation to systemic fibrate clearance, it is essential to quantify phase I and phase II metabolism of fibrates using the same methods and detection platform.

Microsomes have been widely used in enzyme kinetic studies to predict hepatic clearance. Much attention has been placed on the optimization of in vitro incubation conditions [18-20]. However, the in vitro-in vivo extrapolation generally underestimates the metabolism of agents metabolized by multiple pathways [21,22]. One reason for this is that the phase I and phase II metabolic pathways are investigated individually. Recently, it has been proposed that the P450- and UGT-mediated metabolic pathways could be examined simultaneously by adding specific cofactors for compounds metabolized by parallel metabolic pathways $[19,20,23]$. This model may help us to better estimate the metabolism of compounds metabolized by multiple pathways in in vitro-in vivo extrapolation studies.

In the present study, we investigated the metabolism of Gem, CA, FA and Beza using pooled rat liver microsomes (RLM) and human liver microsomes (HLM) based on the same detection platform with the substrate depletion approach. We found that both P450s and UGTs substantially contributed to the clearance of Gem and CA, with UGTs playing a greater role. Although the relative contribution of both pathways for these fibrates in RLM and HLM were similar, the depletion rate of Gem and CA in RLM was higher than that in HLM. These data suggest that RLM can be used to investigate the metabolism of fibrates in humans, but the similarities as well as the differences should be considered when interpreting the results.

\section{Materials and methods}

\subsection{Chemicals and reagents}

UDPGA, alamethicin, saccharic acid lactone, CA and Beza were purchased from Sigma-Aldrich (St. Louis, MO, USA). NADPH and HLM were obtained from BD Gentest (Woburn, MA, USA). FA was purchased from Shangqiu Chemry Chemicals Co. (Shangqiu, China). Gem was obtained from Hunan Qianjin Xiangjiang Pharmaceutical Co. Ltd. (Zhuzhou, China). Acetonitrile and methanol were acquired from Burdick and Jackson (Muskeson, MI, USA). Formic acid was purchased from Dima Technology INC. (Richmond Hill, NY, USA). Deionized water was produced using a Milli-Q System (Milford, MA, USA). All other reagents and chemicals were of analytical grade from commercial sources.

\subsection{Preparation of RLM}

Pooled RLM were prepared as previously described with minor modifications [24,25]. Briefly, 20 livers of Sprague-Dawley rats (10 female and 10 male) were dissected after perfusion and homogenized. The resulting solutions were centrifuged twice at $9000 \times g$ for $20 \mathrm{~min}$. Microsomes were isolated by centrifugation at $100000 \times g$ for $60 \mathrm{~min}$. The precipitant was reconstituted in phosphate buffer and stored at $-80^{\circ} \mathrm{C}$ until use. The protein concentration of microsomes was determined using a Lowry assay.

\subsection{Metabolism of fibrates in RLM}

All incubations were conducted in a shaking water bath at $37^{\circ} \mathrm{C}$ and performed at least in duplicate. The substrate concentration was optimized in preliminary experiments, allowing analytical measurement of rapidly metabolized substrates. Various concentrations of the fibrates were incubated with RLM in a $1.5-\mathrm{mL}$ reaction system. The final concentration of organic solvent in reaction systems did not exceed $0.5 \%$. Reactions were terminated after incubation for $0,2,4,8,12,20,30$ or $40 \mathrm{~min}$ by transferring $0.1 \mathrm{~mL}$ of the mixture to $0.2 \mathrm{~mL}$ of ice-cold acetonitrile containing an internal standard (IS). The terminated solutions were vortexed and the supernatant was separated by centrifugation at $16000 \times g$ for $20 \mathrm{~min}$ at $4^{\circ} \mathrm{C}$. Aliquots $(20 \mu \mathrm{L})$ of the supernatant were injected into the LC-MS/MS system for analysis.

(i) Phase I metabolism. The pooled RLM were diluted in $0.1 \mathrm{~mol} / \mathrm{L}$ phosphate buffer (pH 7.4, EDTA $1 \mathrm{mmol} / \mathrm{L})$. Fibrates were added to final concentrations of 1, 6, 20, 40 and $80 \mu \mathrm{mol} / \mathrm{L}$ for Gem, 0.005, 0.05, 0.5, 2, and $10 \mu \mathrm{mol} / \mathrm{L}$ 
for CA. Because Beza and FA undergo less metabolism before clearance $[11,26]$, accurate measurement of their elimination was difficult. For this reason, we prepared two low concentrations $(0.1$ and $1 \mu \mathrm{mol} / \mathrm{L})$ of Beza and FA and obtained samples at three times (0,20 and $40 \mathrm{~min})$. After 5 min pre-incubation at $37^{\circ} \mathrm{C}$, the reactions were initiated by adding NADPH to a final concentration of $1 \mathrm{mmol} / \mathrm{L}$. The reactions were stopped and quantified as described above.

(ii) Phase II metabolism. Pooled RLM were diluted in $0.1 \mathrm{~mol} / \mathrm{L}$ phosphate buffer $(\mathrm{pH} 7.4$, EDTA $1 \mathrm{mmol} / \mathrm{L})$ containing $D$-saccharic acid 1,4-lactone $(5 \mathrm{mmol} / \mathrm{L})$ and $\mathrm{MgCl}_{2}(5 \mathrm{mmol} / \mathrm{L})$. The diluted microsomes were activated by alamethicin $(0.05 \mathrm{mg} / \mathrm{mg}$ microsomal protein $)$ and placed on ice for $15 \mathrm{~min}$. Gem $(2,15,50,150$ and 500 $\mu \mathrm{mol} / \mathrm{L}), \mathrm{CA}(0.005,0.05,0.5,2$ and $10 \mu \mathrm{mol} / \mathrm{L})$, FA $(0.1$ and $1 \mu \mathrm{mol} / \mathrm{L})$ and Beza $(0.1$ and $1 \mu \mathrm{mol} / \mathrm{L})$, together with activated microsomes, were pre-incubated at $37^{\circ} \mathrm{C}$ for $5 \mathrm{~min}$. Reactions were started by adding UDPGA cofactor (5 $\mathrm{mmol} / \mathrm{L})$. The other procedures were performed as described above for phase I metabolism.

(iii) Combined phase I and phase II metabolism. The pooled RLM and drug solutions were treated as described above for glucuronidation. The reactions were started by adding NADPH ( $1 \mathrm{mmol} / \mathrm{L})$ and UDPGA $(5 \mathrm{mmol} / \mathrm{L})$ together. The other procedures were performed as described above for phase I metabolism.

\subsection{Comparison of metabolism in RLM and HLM}

The metabolic clearance of fibrates in RLM and HLM was compared in the above three conditions using two concentrations of each fibrate (Gem: 2 and $50 \mu \mathrm{mol} / \mathrm{L}$; CA: 0.005 and $0.5 \mu \mathrm{mol} / \mathrm{L}$; Beza: 0.05 and $1 \mu \mathrm{mol} / \mathrm{L}$; FA: 0.05 and 1 $\mu \mathrm{mol} / \mathrm{L})$. The reactions were terminated at $0,4,8,15,30$ and $45 \mathrm{~min}$.

\subsection{LC-MS/MS assay}

Analytes were quantified by liquid chromatography-mass spectrometry (LC-MS) consisting of an high-performance liquid chromatography system (HPLC-10ATVP, Shimadzu, Kyoto, Japan) and Biosystems API 3000 triple-quadrupole mass spectrometer equipped with an electrospray ionization source (Applied Biosystems, Concord, Canada). The MS conditions were optimized and detection was performed using multiple reactions monitored in the negative mode. The $\mathrm{m} / \mathrm{z}$ transition was $249.1 / 121.1$ for Gem, 213.1/126.9 for CA, 317.1/231.1 for FA, and 360.1/274.3 for Beza. Because of their similar scaffolds (Figure 1), CA was used as the IS for Beza, while Beza was used for the other fibrates. The remaining substrates were quantified by comparing peak area ratios (analyte/IS).

To analyze CA, FA and Beza by chromatography, $\mathrm{CH}_{3} \mathrm{OH} / \mathrm{H}_{2} \mathrm{O}$ (10/90) containing $0.1 \%$ formic acid was used as mobile phase A and $\mathrm{CH}_{3} \mathrm{OH} / \mathrm{H}_{2} \mathrm{O}(90 / 10)$ containing
$0.1 \%$ formic acid was used as mobile phase B. The flow rate was kept at $0.2 \mathrm{~mL} / \mathrm{min}$. An HPLC column Capcell Pak C18 $(5 \mu \mathrm{m}, 20 \mathrm{~mm}$ (i.d.) $\times 50 \mathrm{~mm})$ equipped with an ODS guard column $(4 \mathrm{~mm} \times 2.0 \mathrm{~mm}$ (i.d.)) was used. The mobile phase gradient was set as follows: 0 to $0.1 \mathrm{~min}, 10 \% \mathrm{~B} ; 0.1$ to $1.5 \mathrm{~min}$, gradient to $60 \% \mathrm{~B} ; 1.5$ to 1.8 , gradient to $100 \%$ $\mathrm{B}$; 1.8 to $3.0 \mathrm{~min}, 100 \% \mathrm{~B}$; followed by a gradient to $10 \% \mathrm{~B}$ at full speed through to $4.5 \mathrm{~min}$. A Capcell Pak C18 column $(5 \mu \mathrm{m}, 2.0 \mathrm{~mm}$ (i.d.) $\times 100 \mathrm{~mm}$ ) was used to separate $\mathrm{Gem}$ and its metabolites. The HPLC gradient program was optimized as follows: 0 to $1 \mathrm{~min}, 20 \% \mathrm{~B}$; 1 to $1.4 \mathrm{~min}$, gradient to $85 \% \mathrm{~B} ; 1.4$ to $4.0 \mathrm{~min}$, gradient to $100 \% \mathrm{~B} ; 4.0$ to 5.5 $\min , 100 \% \mathrm{~B}$; and then, gradient to $20 \% \mathrm{~B}$ at full speed through to $8 \mathrm{~min}$.

Validation confirmed that this LC-MS/MS assay method met our needs for metabolite quantification. The analytes and the ISs were fully separated, as shown in Figure 2. The signal/noise ratios exceeded 10 and the relative standard deviation (RSD) of triplicate samples were below $15 \%$. After normalization, the percent of substrate remaining was calculated by setting the initial concentration as $100 \%$.

\subsection{Data analysis}

Data acquisition was performed using Analyst 1.4.2 software (Applied Biosystems, Foster City, CA, USA). The
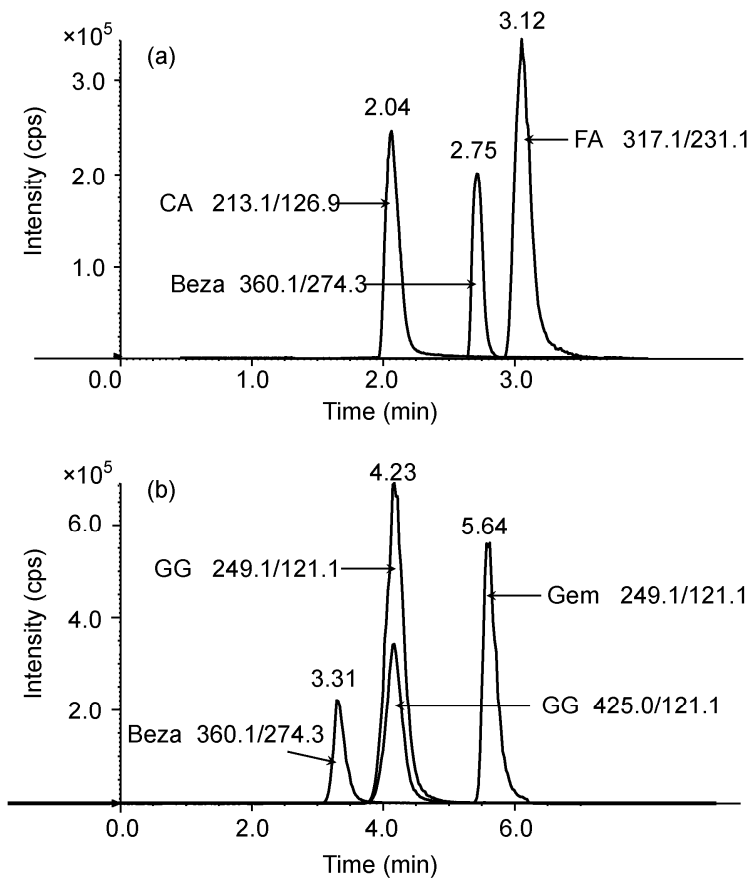

Figure 2 Typical MRM chromatograms for the analytes and internal standards. (a) Typical multiple reaction monitoring (MRM) chromatograms for bezafibrate (Beza), fenofibric acid (FA) and clofibric acid (CA) (negative mode). The transition $\mathrm{m} / \mathrm{z}$ values and elution times were 213.1/126.9 and 2.04 min for CA, 360.1/274.3 and $2.75 \mathrm{~min}$ for Beza, and 317.1/231.1 and $3.12 \mathrm{~min}$ for FA. (b) Typical MRM chromatograms for gemfibrozil (Gem) (negative mode). The transition $\mathrm{m} / \mathrm{z}$ values and elution times were 360.1/274.3 and 3.31 min for Beza, 425.0/121.1, 249.1/121.1 and 4.23 min gemfibrozil glucuronide (GG), and 249.1/121.1 and $5.64 \mathrm{~min}$ for Gem. 
apparent enzyme kinetic parameters were determined by the substrate depletion approach. The percentage remaining versus time at each substrate concentration was fitted with a first-order decay curve to calculate the apparent rate constant for substrate depletion $\left(K_{\mathrm{dep}}\right)$. If later times showed nonlinearity, only the initial times were used to determine $K_{\text {dep }}[25]$.

$K_{\mathrm{dep}([S] \rightarrow 0)}$ and $K_{\mathrm{m}}$ parameters were determined by fitting the $K_{\text {dep }}$ and $[S]$ to eq. (1) proposed by Obach and ReedHagen [27] with Origin software (version 7.5, MicroCal Software, Inc., Piscataway, NJ, USA).

$$
K_{\mathrm{dep}}=K_{\mathrm{dep}([s] \rightarrow 0)}\left(1-\frac{[S]}{[S]+K_{\mathrm{m}}}\right) .
$$

In this experimental equation, $[S]$ and $K_{\mathrm{m}}$ are the substrate concentration and Michaelis constant, respectively, and $K_{\text {dep }([S] \rightarrow 0)}$ represents the theoretical $K_{\text {dep }}$ at the infinitesimal substrate concentration. The maximum velocity of enzyme $\left(V_{\max }\right)$ was calculated using eq. (2) and intrinsic clearance $\left(C L_{\text {int }}\right)$ was calculated using eq. (3) [28].

$$
\begin{gathered}
V_{\max }=K_{\operatorname{dep}([s] \rightarrow 0)} K_{\mathrm{m}}, \\
C L_{\text {int }}=\frac{V_{\max }}{K_{\mathrm{m}}} .
\end{gathered}
$$

\section{Results}

\subsection{Metabolism of fibrates in RLM}

P450- and UGT-mediated metabolism of fibrates was measured by applying the cofactors NADPH and UDPGA either individually or together. The depletion profiles of
Gem and CA are plotted in Figure 3. Both Gem and CA were eliminated in a linear manner at all concentrations. The percentage of remaining substrates versus time for each concentration was successfully fitted with a first-order decay curve to calculate the apparent $K_{\mathrm{dep}}$-the slope of each substrate concentration curve.

P450- and UGT-mediated metabolism of FA and Beza was also examined in RLM. The relative contributions of each pathway to their clearance were markedly less for FA and Beza compared with Gem and CA, which is similar to previous reports $[11,26]$. Thus it is difficult to quantitatively measure substrates showing rapid metabolism at high substrate concentrations, and the intrinsic clearance of both substrates was too low to be determined using the substrate depletion approach. Therefore, we tested two low concentrations $(0.1$ and $1 \mu \mathrm{mol} / \mathrm{L})$, and the results are shown in Figure 4 . The results indicated that the contributions of the phase I and phase II pathways to Beza metabolism were not significant at the two concentrations tested. Phase II metabolism had a greater contribution than phase I metabolism to the metabolic clearance of FA, although its rate of depletion was slow.

$K_{\text {dep }}$ values were successfully determined using the five concentrations for Gem and CA by fitting the percentage of substrate remaining versus time at each initial concentration with a first-order decay curve. The apparent enzyme kinetic parameters for Gem and CA were calculated using eqs. (1)-(3), and the values are shown in Table 1.

The $K_{\mathrm{m}}, V_{\max }$ and $C L_{\text {int }}$ of Gem in P450-mediated metabolism were $11 \pm 4 \mu \mathrm{mol} / \mathrm{L}, 1861 \pm 967 \mathrm{nmol} \mathrm{min}^{-1} \mathrm{mg}^{-1}$ and $172 \pm 22 \mu \mathrm{L} \mathrm{min}{ }^{-1} \mathrm{mg}^{-1}$, respectively. The $K_{\mathrm{m}}$ of Gem for glucuronidation $(11 \pm 1 \mu \mathrm{mol} / \mathrm{L})$ was similar to the $K_{\mathrm{m}}$ for the phase I oxidation reaction, indicating similar affinities of both enzyme groups for Gem. However, the $V_{\max }$ for
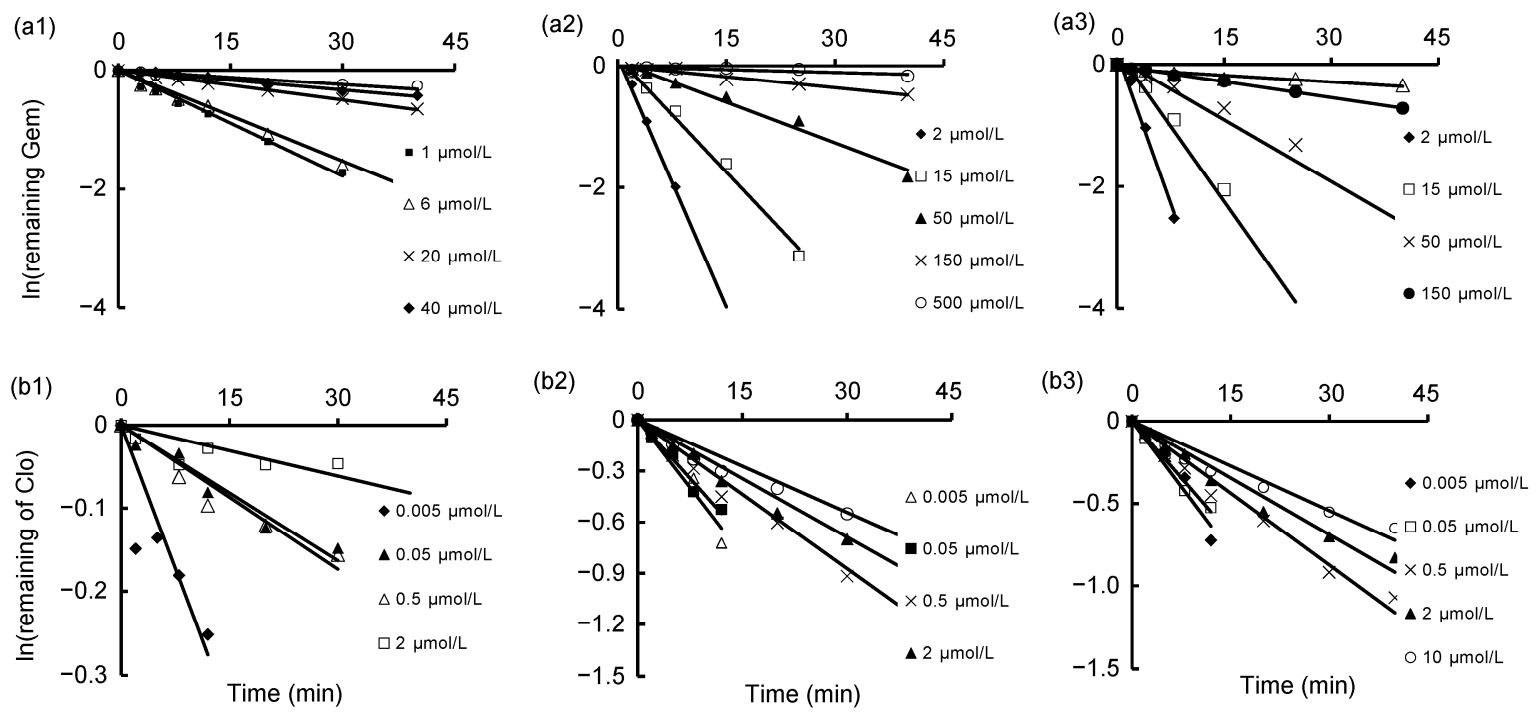

Figure 3 Elimination rate constant-time profiles of Gem (a) and CA (b) in RLM. The $Y$-axis represents the logarithmic value of the remaining percentage of substrate relative to the initial concentration. (a1, b1) P450-mediated metabolism; (a2, b2) UGT-mediated metabolism; (a3, b3) P450- and UGT-mediated metabolism. Gem: gemfibrozil; CA: clofibric acid. 

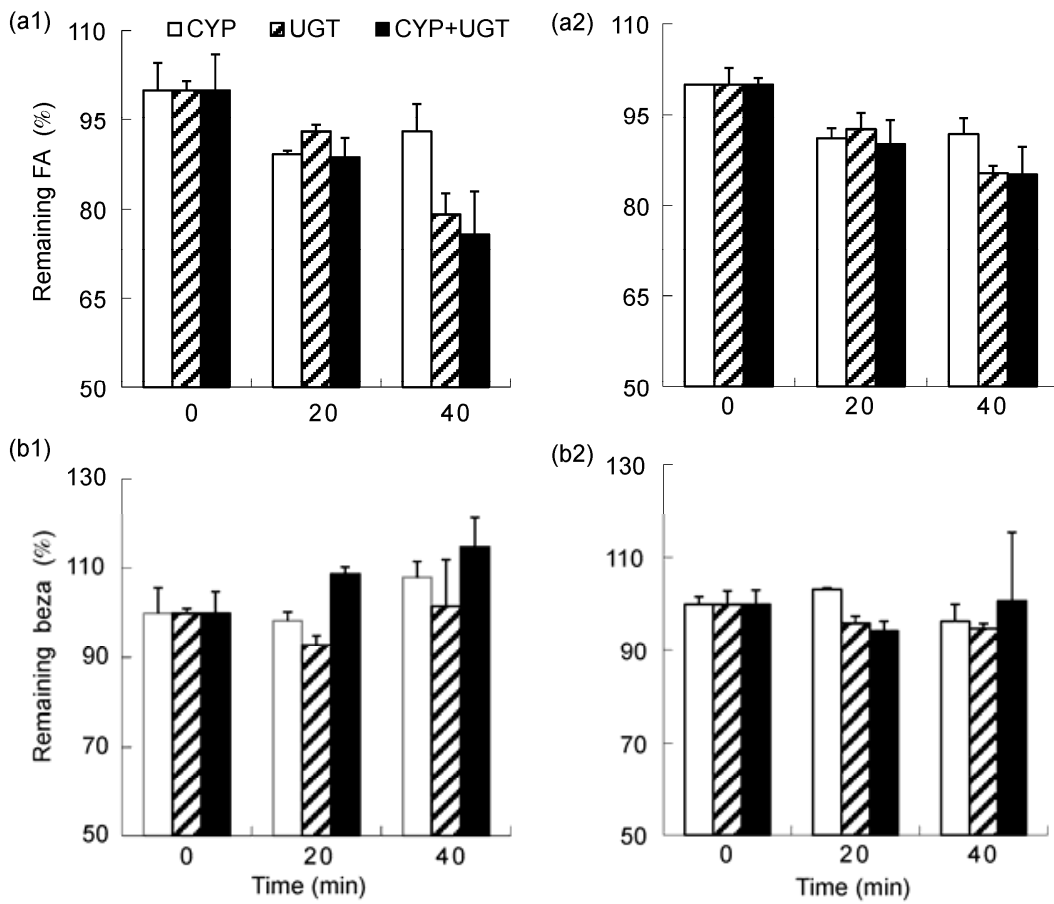

Figure 4 Elimination profiles of fenofibric acid (a) and bezafibrate (b) in RLM. Substrates were tested at either 0.1 (a1, b1) or 1 (a2, b2) $\mu \mathrm{mol} / \mathrm{L}$.

Table 1 Enzyme kinetics and the estimated $F m_{(\mathrm{P} 450)}$ and $F m_{(\mathrm{UGT})}$ values for gemfibrozil and clofibric acid in RLM ${ }^{\text {a) }}$

\begin{tabular}{ccccccc}
\hline Drug & Reactions & $K_{\mathrm{m}}(\mu \mathrm{mol} / \mathrm{L})$ & $V_{\max }\left(\mathrm{nmol} \mathrm{min} \mathrm{mg}^{-1}\right)$ & $C L_{\text {int }}\left(\mu \mathrm{L} \mathrm{min}^{-1} \mathrm{mg}^{-1}\right)$ & $F m_{(\mathrm{P} 450)}{ }^{*}$ & $F m_{(\mathrm{UGT})} * *$ \\
\hline \multirow{3}{*}{ Gemfibrozil } & P450 & $11 \pm 4$ & $1861 \pm 967$ & $172 \pm 22$ & & \\
& UGT & $11 \pm 1$ & $6993 \pm 1183$ & $643 \pm 26$ & $22 \%$ & $81 \%$ \\
\hline \multirow{3}{*}{ Clofibric acid } & P450+UGT & $8 \pm 3$ & $6100 \pm 3041$ & $798 \pm 103$ & & \\
& P450 & $0.04 \pm 0.004$ & $2.3 \pm 0.3$ & $43 \pm 11$ & & \\
& UGT & $2 \pm 1$ & $164 \pm 113$ & $88 \pm 12$ & $36 \%$ & $74 \%$ \\
\hline
\end{tabular}

a) $*, F m_{(\mathrm{P} 450)}=C L_{\text {int }(\mathrm{P} 450)} / C L_{\text {int }(\mathrm{P} 450+\mathrm{UGT})} ; * *, F m_{(\mathrm{UGT})}=C L_{\text {int }(\mathrm{UGT})} / C L_{\text {int }}(\mathrm{P} 450+\mathrm{UGT})$.

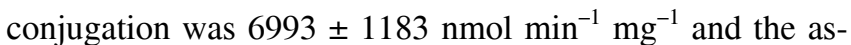
sociated $C L_{\text {int }}$ was $643 \pm 26 \mu \mathrm{L} \mathrm{min}{ }^{-1} \mathrm{mg}^{-1}$, which was much higher than the corresponding value for oxidation. Therefore, the phase II pathway is much more important than the phase I pathway for Gem metabolism. When applying both cofactors simultaneously, the $K_{\mathrm{m}}$ and $V_{\max }$ values were $8 \pm 3 \mu \mathrm{mol} / \mathrm{L}$ and $6100 \pm 3041 \mathrm{nmol} \mathrm{min}{ }^{-1} \mathrm{mg}^{-1}$, respectively, and the $C L_{\text {int }}$ was $798 \pm 103 \mu \mathrm{L} \mathrm{min}{ }^{-1} \mathrm{mg}^{-1}$, which was nearly equal to the sum of the individual values for oxidation and glucuronidation.

The $K_{\mathrm{m}}, V_{\max }$, and $C L_{\text {int }}$ values for CA via the $\mathrm{P} 450$ mediated metabolic pathway were $0.04 \pm 0.004 \mu \mathrm{mol} / \mathrm{L}, 2.3$ $\pm 0.3 \mathrm{nmol} \mathrm{min} \mathrm{mg}^{-1}$ and $43 \pm 11 \mu \mathrm{L} \mathrm{min} \mathrm{mg}^{-1}$, respectively. The $K_{\mathrm{m}}$ for CA via UGT-mediated glucuronidation was $2 \pm 1 \mu \mathrm{mol} / \mathrm{L}$, which was much higher than that for phase I oxidation. However, the $V_{\max }$ for conjugation was $164 \pm 113 \mathrm{nmol} \mathrm{min}^{-1} \mathrm{mg}^{-1}$ and the associated $C L_{\text {int }}$ was 88 $\pm 12 \mu \mathrm{L} \mathrm{min}{ }^{-1} \mathrm{mg}^{-1}$, which was much higher than that for oxidation. These results suggest that the lower $V_{\max }$ for phase I metabolism reduced its contribution associated with higher affinity substrates. Thus, glucuronidation contributed more than oxidation to CA metabolism. When applying both cofactors simultaneously, the $C L_{\text {int }}$ was $119 \pm 13 \mu \mathrm{L}$ $\mathrm{min}^{-1} \mathrm{mg}^{-1}$, which was nearly equal to the sum of the individual values for oxidation and glucuronidation.

The fractions of Gem metabolized by NADPH-dependent oxidation $\left(F m_{(\mathrm{P} 450)}\right)$ and UDPGA-dependent glucuronidation $\left(F m_{\text {(UGT) }}\right)$ were $22 \%$ and $81 \%$, respectively. For CA, $F m_{(\mathrm{P} 450)}$ and $F m_{\text {(UGT) }}$ were $36 \%$ and $74 \%$, respectively. These data suggest that metabolism of Gem and CA displayed NADPH and UDPGA dependency, and that individual measurement of NADPH-dependent or UDPGA-dependent pathways in isolation does not represent the actual metabolism of fibrates. Accordingly, it is necessary to monitor both NADPH- and UDPGA-dependent metabolism for drugs metabolized by multiple pathways. 


\subsection{Differences in Gem and CA metabolism between RLM and HLM}

Using the same methods, we examined the metabolism of all four fibrates at two concentrations in both RLM and HLM. As in RLM, the metabolism of Beza and FA could not be accurately determined in HLM (data not shown). Therefore, we did not compare the metabolic profiles of RLM and HLM for Beza and FA. In contrast, we were able to compare the metabolism of Gem and CA between RLM and HLM and the resulting profiles are shown in Figure 5.

Interestingly, the relative contribution of glucuronidation versus oxidation to Gem metabolism was similar in RLM and HLM. The rates of Gem depletion by P450-mediated oxidation and UGT-mediated glucuronidation were 303 and $1607 \mathrm{nmol} \mathrm{min}{ }^{-1} \mathrm{mg}^{-1}$, respectively, in RLM versus 85.5 and $243 \mathrm{nmol} \mathrm{min} \mathrm{mg}^{-1}$, respectively, in HLM. For CA, $\mathrm{P} 450$-mediated metabolic clearance was not obvious in either RLM or HLM (Figure 5(b1)); however, the rate of CA depletion by the UGT-mediated pathway was higher in RLM than in HLM (Figure 5(b2)). The UGT-mediated pathway exerted a much greater contribution to the metabolism of FA than did the P450-mediated pathway (Figure 4(a1)). These data indicate that Gem, CA and FA are metabolized by similar pathways in RLM and HLM, and glucuronidation plays a more important role than oxidation in their metabolism.

However, some species differences between rats and human were apparent in our data in term of the rate of depletion. The depletion rate of Gem by oxidation was 303 nmol min ${ }^{-1} \mathrm{mg}^{-1}$ in RLM versus $85.5 \mathrm{nmol} \mathrm{min} \mathrm{mg}^{-1}$ in HLM, while the corresponding rates for glucuronidation were $1607 \mathrm{nmol} \mathrm{min}{ }^{-1} \mathrm{mg}^{-1}$ versus $243 \mathrm{nmol} \mathrm{min}{ }^{-1} \mathrm{mg}^{-1}$. Meanwhile, the rate of depletion of CA by the UGTmediated pathway was higher in RLM than in HLM (Figure 5(b2)). Taken together, these data suggest that the metabolic clearance of Gem and CA was higher in RLM than that in HLM, when mediated by oxidation, glucuronidation or both.

\section{Discussion}

The metabolism of fibrates is known to involve multiple pathways, but the results published to date differ in terms of the relative contributions of each pathway. This may be partly due to the fact that the contributions of the major metabolic pathways to fibrate clearance have not been systematically investigated. Previous in vitro studies generally focused on a specific pathway by adding either NADPH or UDPGA to initiate oxidative or conjugated reactions [22,29]. Such strategies may hence underestimate the true metabolic rate of fibrates when using in vitro-in vivo extrapolation studies because the contribution of both pathways should not be ignored. In this study, we measured the metabolism of fibrates in RLM and HLM by applying the cofactors NADPH and UDPGA individually and simultaneously, allowing us to determine the contributions of both pathways using the same approach and detection platform. The substrate depletion approach has been shown to be comparable (a1)

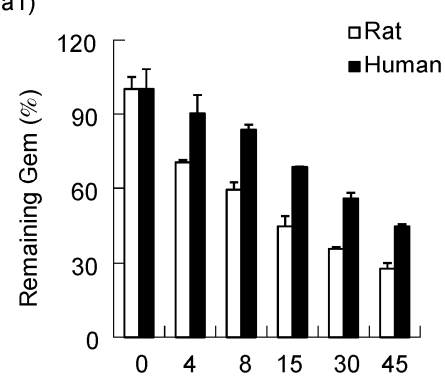

(b1)

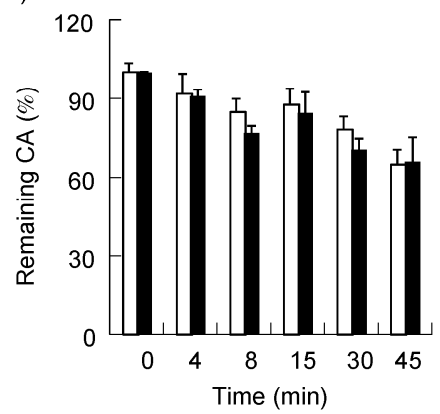

(a2)

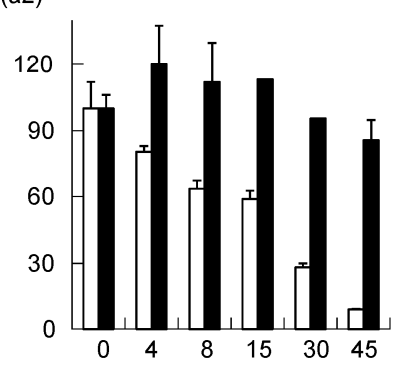

(b2)

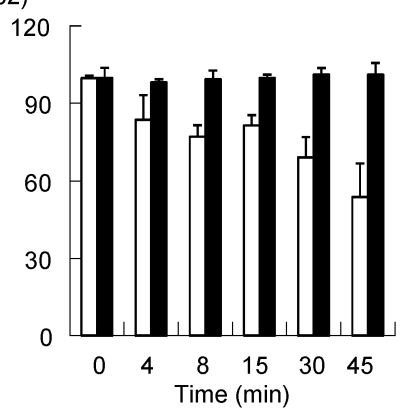

(a3) 120

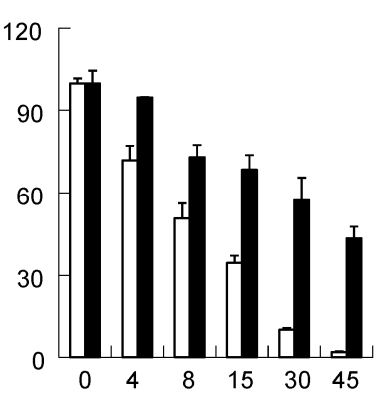

(b3)

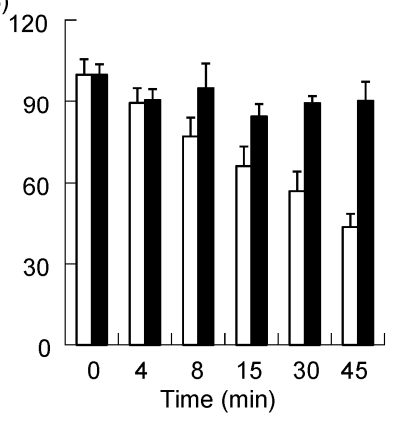

Figure 5 Comparison of the elimination profiles of Gem (a) and CA (b) in RLM (unpacked column) and HLM (packed column). (a1, b1) P450-mediated metabolism; (a2, b2) UGT-mediated metabolism; (a3, b3) P450- and UGT-mediated metabolism. Gem: gemfibrozil; CA: clofibric acid. 
to the product formation approach and is increasingly being used in enzyme kinetics studies [23,27,30]. Considering the diversity of metabolites formed from the metabolism of fibrates and the requirement for semi throughput in this study, it is impractical to monitor product formation; thus the substrate depletion approach is more appropriate in this context.

In this study, Gem was metabolized by glucuronidation and oxidation, although glucuronidation played a much greater role than oxidation in both RLM and HLM. Biotransformation of CA also displayed NADPH and UDPGA dependency in RLM, with a much greater role of UGTmediated glucuronidation. Similarly, FA was predominantly cleared in RLM via UGT-mediated glucuronidation. These data are consistent with those reported in clinic [11,13-15]. Our study showed that both P450s and UGTs are involved in fibrate metabolism. Therefore, measurement of either NADPH-dependent or UDPGA-dependent pathways in isolation may underestimate the true rate of clearance of fibrates. Therefore, it is important to examine both pathways when assaying the metabolism of substrates metabolized via multiple pathways.

In terms of the metabolic profiles of fibrates examined in this study, we found some similarities and some differences between RLM and HLM. Gem and CA were principally eliminated through conjugative routes in both RLM and HLM, with smaller contributions from P450s. These data resembled those of a previous study in a clinical setting [11]. Taking Gem as an example, the contribution of glucuronidation to its metabolic clearance was $81 \%$, which is similar to previously reported value of $79 \%$ in HLM [20]. Similarly, the contribution of glucuronidation to CA clearance was much more important than oxidation in both RLM and HLM. However, different rates of depletion of these fibrates were detected between RLM and HLM. As shown in Figure 5, the P450- and UGT-mediated depletion rates for Gem and CA were much higher in RLM than in HLM. Clearly, such relationships and differences should be considered when extrapolating between these two species.

In conclusion, the P450 and UGT pathways substantially contributed to the clearance of Gem and CA. To avoid underestimating the rates of clearance, it is necessary to measure both NADPH- and UDPGA-dependent metabolism for drugs metabolized by multiple pathways. Although the relative fractions of both pathways for Gem and CA were similar, glucuronidation played a much greater role in both RLM and HLM. Our findings suggest that RLM are suitable model to examine fibrate metabolism in humans, providing differences in the rate of depletion between RLM and HLM are taken into account.

This work was supported by the Key Program for International S\&T Cooperation Projects of China (2008AA02Z314 and 2009ZX09301-015), Zhejiang Provincial Natural Science Foundation of China (Y2110016), and Ningbo Natural Science Foundation (2011A610059).
1 Thorp J M. Experimental evaluation of an orally active combination of androsterone with ethyl chlorophenoxyisobutyrate. Lancet, 1962, 1: 1323-1326

2 Elsom L F, Hawkins D R, Chasseaud L F. Identification of a major metabolite of new hypolipemic agent, isopropyl 2-4'( $p$-chlorobenzoyl) phenoxy-2-methylpropionate (procetofene) in humans by gas chromatography mass spectrometry. J Chromatogr, 1976, 123: 463-467

3 Desager J P, Hulhoven R, Harvengt C. Uricosuric effect of fenofibrate in healthy-volunteers. J Clin Pharmacol, 1980, 20: 560-564

4 Reddy J K, Azarnoff D L, Hignite C E. Hypolipemic hepatic peroxisome proliferators form a novel class of chemical carcinogens. Nature, 1980, 283: 397-398

5 Rao M S, Reddy J K. Peroxisome proliferation and hepatocarcinogenesis. Carcinogenesis, 1987, 8: 631-636

6 Klaunig J E, Babich M A, Baetcke K P, et al. PPAR alpha agonist-induced rodent tumors: Modes of action and human relevance. Crit Rev Toxicol, 2003, 33: 655-780

7 Blane G F. Comparative toxicity and safety profile of fenofibrate and other fibric acid-derivatives. Am J Med, 1987, 83: 26-36

8 Lupp A, Karge E, Hopf H, et al. Fibrates and their newly synthesized glycinate or glycinate-methylester derivatives: Comparison of the interactions with liver cytochrome P450 dependent monooxygenaseand oxidase-functions in vitro. Exp Toxicol Pathol, 2003, 54: 433440

9 Ogilvie B W, Zhang D L, Li W Y, et al. Glucuronidation converts gemfibrozil to a potent, metabolism-dependent inhibitor of CYP2C8: Implications for drug-drug interactions. Drug Metab Dispos, 2006, 34: 191-197

10 Miller D B, Spence J D. Clinical pharmacokinetics of fibric acid derivatives (fibrates). Clin Pharmacokinet, 1998, 34: 155-162

11 Cayen M N. Disposition, metabolism and pharmacokinetics of antihyperlipidemic agents in laboratory-animals and man. Pharmacol Therapeut, 1985, 29: 157-204

12 Nakagawa A, Shigeta A, Iwabuchi H, et al. Simultaneous determination of gemfibrozil and its metabolites in plasma and urine by a fully automated high-performance liquid-chromatographic system. Biomed Chromatogr, 1991, 5: 68-73

13 Smith T C. Toleration and bioavailability of gemfibrozil in healthy men. Proc R Soc Med, 1976, 69 (Suppl 2): 24-27

14 Okerholm R A, Keeley F J, Peterson F E, et al. Metabolism of gemfibrozil. Proc R Soc Med, 1976, 69: 11-14

15 Sedaghat A, Ahrens E H Jr. Lack of effect of cholestyramine on the pharmacokinetics of clofibrate in man. Eur J Clin Invest, 1975, 5: 177-185

16 Brodie R R, Chasseaud L F, Elson F F, et al. Antilipidemic drugs. Part 4: The metabolic fate of the hypolipidemic agent isopropyl-[4'(p-chlorobenzoyl)-2-phenoxy-2-methyl]-protionate (LF 178) in rats, dog and man. Arzneimittel-Forschung, 1976, 26: 896-901

17 Oster P, Lang P D, Vollmar J, et al. Diurnal lipid and lipoprotein profiles in hypertriglyceridemic patients with bezafibrate and clofibrate. Res Exp Med, 1986, 186: 435-441

18 Soars M G, Ring B J, Wrighton S A. The effect of incubation conditions on the enzyme kinetics of UDP-glucuronosyltransferases. Drug Metab Dispos, 2003, 31: 762-767

19 Yan Z, Caldwell G W. Metabolic assessment in liver microsomes by co-activating cytochrome P450s and UDP-glycosyltransferases. Eur J Drug Metab Ph, 2003, 28: 223-232

20 Kilford P J, Stringer R, Sohal B, et al. Prediction of drug clearance by glucuronidation from in vitro data: Use of combined cytochrome P450 and UDP-glucuronosyltransferase cofactors in alamethicinactivated human liver microsomes. Drug Metab Dispos, 2009, 37: 82-89

21 Soars M G, Burchell B, Riley R J. In vitro analysis of human drug glucuronidation and prediction of in vivo metabolic clearance. $\mathrm{J}$ Pharmacol Exp Ther, 2002, 301: 382-390

22 Miners J O, Knights K M, Houston J B, et al. In vitro-in vivo correlation for drugs and other compounds eliminated by glucuronidation in humans: Pitfalls and promises. Biochem Pharmacol, 2006, 71: 1531-1539 
23 Mohutsky M A, Chien J Y, Ring B J, et al. Predictions of the in vivo clearance of drugs from rate of loss using human liver microsomes for phase I and phase II biotransformations. Pharm Res, 2006, 23: 654-662

24 Hutson J L, Higgins J A. Reversible activation inactivation of the deacylation acylation cycle in rat-liver microsomes. Bioscience Rep, 1987, 7: 73-80

25 Mei L, Zhang L, Dai R. An inhibition study of beauvericin on human and rat cytochrome $\mathrm{P} 450$ enzymes and its pharmacokinetics in rats. $\mathrm{J}$ Enzym Inhib Med Ch, 2009, 24: 753-762

26 Facino R M, Carini M, Bertuletti R, et al. "In vitro" metabolism of bezafibrate by rat liver. Pharmacol Res Commun, 1981, 13: 121-132
27 Obach R S, Reed-Hagen A E. Measurement of Michaelis constants for cytochrome $\mathrm{P} 450$-mediated biotransformation reactions using a substrate depletion approach. Drug Metab Dispos, 2002, 30: 831-837

28 Nath A, Atkins W M. A theoretical validation of the substrate depletion approach to determining kinetic parameters. Drug Metab Dispos, 2006, 34: 1433-1435

29 Carlile D J, Hakooz N, Bayliss M K, et al. Microsomal prediction of in vivo clearance of CYP2C9 substrates in humans. Br J Clin Pharmacol, 1999, 47: 625-635

30 Jones H M, Houston J B. Substrate depletion approach for determining in vitro metabolic clearance: Time dependencies in hepatocyte and microsomal incubations. Drug Metab Dispos, 2004, 32: 973-982

Open Access This article is distributed under the terms of the Creative Commons Attribution License which permits any use, distribution, and reproduction in any medium, provided the original author(s) and source are credited. 